\title{
A Modified DistFlow for Distributed Generation Planning Problems in Radial Grids
}

\author{
Rémy Rigo-Mariani \\ Univ. Grenoble Alpes, CNRS, Grenoble \\ INP, G2Elab \\ F-38000, Grenoble, France \\ remy.rigo-mariani@grenoble-inp.fr
}

\author{
Vincent Debusschere \\ Univ. Grenoble Alpes, CNRS, Grenoble \\ INP, G2Elab \\ F-38000, Grenoble, France \\ vincent.debusschere@grenoble-inp.fr
}

\author{
Marie-Cécile Alvarez-Herault \\ Univ. Grenoble Alpes, CNRS, Grenoble \\ INP, G2Elab \\ F-38000, Grenoble, France \\ marie-cecile.alvarez-herault@univ-grenoble- \\ alpes.fr
}

\begin{abstract}
This paper focuses on a simplified expression of the load flow constraints for the optimal planning of distributed generation (DG) in radial grids. The proposed approach lies on the conventional DistFlow equations improved with the consideration of linearized bi-directional losses and their impact on the voltage profiles. The method is validated on various radial test cases and allows to run time coupled power flows in order to consider dynamic constraints (e.g. battery state of chare) in short computational times. With the help of the proposed method, a DG placement problem is solved in less than 2 minutes with an exhaustive search over a 69 buses system and the operation is simulated along a single representative day. A scalability test is finally performed with longer time horizons and greater numbers of connected DG. The developed approach allows to run the operation of a 69 buses system over 2 months and with 20 connected DGs in less than 5 minutes.
\end{abstract}

Keywords-Optimal Power Flow, DistFLow, Linearization, Optimal Planning

\section{NOMENCLATURE}

Sets :

$t \in T$

$b \in B$

$l \in L$

set of time steps

set of buses ( $B$ buses)

$k \in K$

set of branches ( $L$ lines)

$l_{d(b)} \in D(b)$

$l_{u(b)}$

$b_{d(l)}$

$b_{u(l)}$

Variables (in p.u.):

set of piece wise segments ( $K$ segments)

set of lines downstream a bus $b$

upstream line of bus $b$ (one element)

downstream/end bus of line $l$ (one element)

upstream/start bus of line $l$ (one element)

For $l=l_{d(b)}$ or $l_{u(b)}$

$p_{l, t}, q_{l, t} \quad$ active and reactive powers in line $l$ at time $t$

$\delta p_{l, t}, \delta q_{l, t} \quad$ active and reactive powers in line $l$ at time $t$

$V_{b, t}$

$p_{l, k, t}^{+}, p_{l, k, t}^{-}$

$q_{l, k, t}^{+}, q_{l, k, t}^{-}$

$\delta p_{l, t}^{+}, \delta p_{l, t}^{-}$

$\delta q_{l, k, t}^{+}, \delta q_{l, k, t}^{-}$ voltage at bus $b$ at time $t$

$P_{b, t}, Q_{b, t}$ $P_{b, t}^{b a t+}, P_{b, t}^{b a t-}$

$S O C_{b, t}^{b a t}$

line $l$ active (+/-) power in block $k$ at time $t$

line $l$ reactive (+/-) power in block $k$ at time $t$

line $l$ active losses (+/-) in line $l$ at time $t$

line $l$ reactive losses (+/-) in line $l$ at time $t$

active and reactive injected at bus $b$ at time $t$

charge/discharge of battery at bus $b$ at time $t$

state of charge of battery at bus $b$ at time $t(\%)$

Parameters :

$P_{b, t}^{L}, Q_{b, t}^{L}$

active and reactive load at bus $b$ at time $t$

$\bar{p}_{l}, \bar{q}_{l}$

line $l$ active reactive limit power

$r_{l}, x_{l}$

$E_{b}^{b a t}, \eta_{b}^{b a t}$

$P_{b, t}^{P V}$

resistance and reactance of line $l$

battery capacity at bus $b$

solar generation at bus $\mathrm{b}$ at time $t$

\section{INTRODUCTION}

The benefits of distributed generation (DG) integrated in conventional power systems have long been identified - with losses and emissions reduction, improved voltage profiles and enhanced system reliability as well as the opportunity to defer significant grid investments [1]. Oftentimes, in the framework of DG planning studies, the objective is to find the best type, location and size of the considered resources that may be renewable based or fossil fueled generators and energy storage units [2]. One of the main technical challenges encountered is to avoid prohibitive computational times. Indeed, when integrated design and management problems are investigated, the operation of the systems has to be simulated for a great number of configurations before finding the optimal DG plan [3]. Especially, when storage devices are considered, the system operation is estimated over a representative period (day, month, year). With energy constraints linked across the successive time steps, the power flow equations have to be simulated over the whole horizon, and typical AC power flow (ACPF) cannot be run successively for every time step, as seen in [4]. One way to leverage that complexity is to consider DC power flow (DCPF) constraints [5] at the cost of extensive simplifications that are not relevant for distribution networks, to which most of the small distributed resources are expected to be connected. In particular, reactive power and voltage drop are not considered. For radial systems, a simplified DistFlow approach allows to efficiently compute the active/reactive power flows while updating the voltage drop form the slack bus (typically the point of common coupling, PCC) to the termination node of every feeder [6]. Linearized approaches of the DistFlow are encountered in planning studies or in the presence of distributed resources for faster computation [7] [8]. Similarly to the DCPF equations, those approaches lie on strong assumptions, the line losses being oftentimes neglected when computing the branch flow or bus voltages. This paper proposes a modified version of the linearized DistFlow to overcome those shortcomings. A particular attention is attached to the consideration of bi-directional active/reactive losses in the context of resources allocation that may incur backfeed power. The method is then validated over various test cases before a generic, simple DG planning problem is considered. The main contribution of the work are:

- A DistFlow formulation that considers linearized bidirectional losses and their impact on the voltage.

- The validation of the method for a simple allocation problem solved in less than 2 min in a 69 bus system.

- A scalability test for longer planning horizons and greater numbers of distributed resources. 


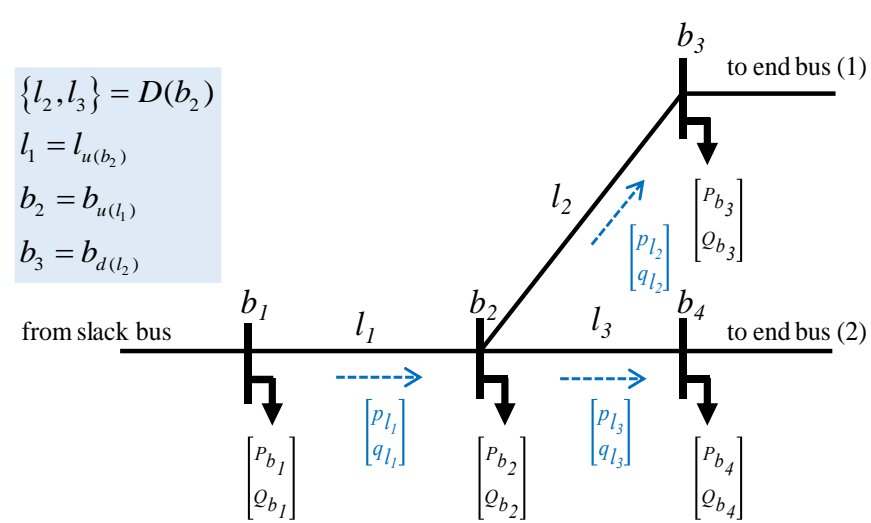

Fig. 1 Single line diagram of a radial network for original DistFlow formulation

\section{MODIFIED DISTFLOW}

\section{A. Classical DistFlow Equations}

The original DistFlow formulation for radial systems is based on the iterative computation of the branch flows and node voltage from the slack bus down to the termination buses of the considered radial network (Fig. 1) [6]. The classical equations in per unit are written while introducing the sets of upstream/downstream lines and buses with (1) and (2) written from every bus $b$ perspective and (3) considered for every line $l$. As already mentioned, the power flow implemented in this paper is intended to simulate scheduled operations over a representative period, which explains the introduction of the temporal set $T$ compared to traditional formulation. Also note that, for radial networks, the set of upstream lines attached to each bus only consists of a single branch. Finally, in the absence of distributed generation (i.e. injection at the slack bus only), the bus injections $P_{b, t}, Q_{b, t}$ are equal to the loads $P_{b, t}^{L}, Q_{b, t}^{L}$.

$$
\begin{aligned}
& \sum_{l_{d(b)} \in D(b)} p_{l_{d(b)}, t}=p_{l_{u(b)}, t}-P_{b, t}-r_{l_{u(b)}} \cdot \frac{p_{l_{u(b)}, t}^{2}+q_{l_{u(b)}, t}^{2}}{V_{b, t}^{2}} \\
& \sum_{l_{d(b)} \in D(b)} q_{l_{d(b)}, t}=q_{l_{u(b)}, t}-Q_{b, t}-x_{l_{u(b)}} \cdot \frac{p_{l_{u(b)}, t}^{2}+q_{l_{u(b)}, t}^{2}}{V_{b, t}^{2}} \\
& V_{b_{d(l)}, t}^{2}=V_{b_{u(l)}, t}^{2}-2 \cdot\left(r_{l} \cdot p_{l, t}+x_{l} \cdot q_{l, t}\right)+\left(r_{l}^{2}+x_{l}^{2}\right) \cdot \frac{p_{l, t}^{2}+q_{l, t}^{2}}{V_{b_{u(l)}, t}^{2}}
\end{aligned}
$$

Typical linearization approaches [7] consist in neglecting the quadratic term that represent the active and reactive line losses in (1) and (2) respectively. At the same time the voltage at each bus is assumed to be close to the reference voltage $V_{0}$. with $\left(V_{b, t}-V_{0}\right)^{2} \sim 0$, which allows (3) to be rewritten following (4) and while neglecting the losses in the computation of the voltage and branch flows. However, the quadratic formulation of the losses is sometimes still considered as the objective function of the considered optimization problems. Similarly to [7] and [8], those losses are then computed somewhat independently, which does not capture their impact on the voltage drops and line flows. The resulting load flow is then a linearly constrained quadratic problem.

$V_{b_{d(l)}, t}=V_{b_{u(l)}, t}-\left(r_{l} \cdot p_{l, t}+x_{l} \cdot q_{l, t}\right) / V_{0}$

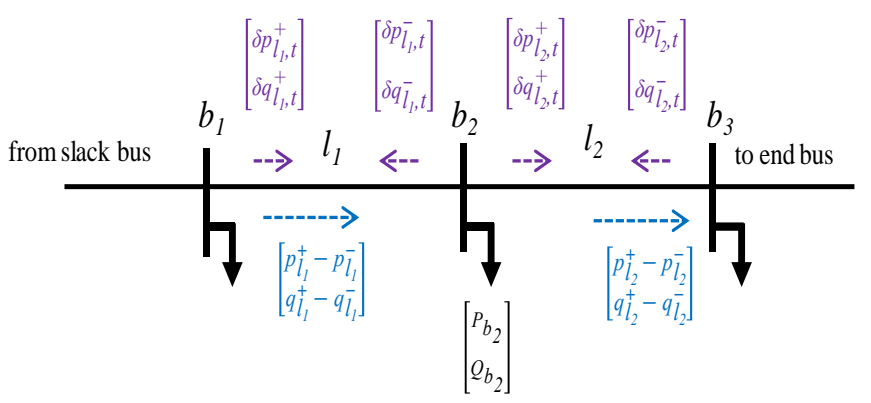

Fig. 2 Introduction of variables for the line losses and bidirectional flows

\section{B. Bi-Directionnal Line Flows and Losses}

In the context of a resources allocation problem, the losses should not be entirely neglected as different solutions (resources location and size) are most of the time discriminated with regard to the system oveall efficiency. In addition, with the connection of distributed generation, lines flow are not necessary unidirectional from the slack bus to the termination nodes. Indeed, local injection at a given bus may result in reverse power flow in the absence of significant enough load consumption. In order to represent potential bidirectional line flows and associated losses, additional semi definite positive variables are introduced in this paper (refer to Fig. 2 and (5)), and the standard DistFlow equations are rewritten following (6)-(8). That formulation specifically allows to estimate the impact of the losses on the bus voltage and line flows, independently for both active and reactive contributions, and for every possible combination of flow directions.

$$
\begin{aligned}
& \left\{\begin{array} { l } 
{ p _ { l , t } = p _ { l , t } ^ { + } - p _ { l , t } ^ { - } } \\
{ q _ { l , t } = q _ { l , t } ^ { + } - q _ { l , t } ^ { - } }
\end{array} \text { and } \left\{\begin{array}{l}
\delta p_{l, t}=\delta p_{l, t}^{+}-\delta p_{l, t}^{-} \\
\delta q_{l, t}=\delta q_{l, t}^{+}-\delta q_{l, t}^{-}
\end{array}\right.\right. \\
& \sum_{l_{d(b)} \in D(b)}\left(p_{l_{d(b)}, t}+\delta p_{l_{d(b)}, t}\right)=p_{l_{u(b)}, t}+\delta p_{l_{u(b)}, t}-P_{b, t} \\
& \sum_{l_{d(b)} \in D(b)}\left(q_{l_{d(b)}, t}+\delta q_{l_{d(b)}, t}\right)=q_{l_{u(b)}, t}+\delta q_{l_{u(b)}, t}-Q_{b, t}^{L} \\
& V_{b_{d(l)}, t}=V_{b_{d(l)}, t}-\left(r_{l} \cdot\left(p_{l, t}+\delta p_{l, t}\right)+x_{l} \cdot\left(q_{l, t}+\delta q_{l, t}\right)\right) / V_{0}
\end{aligned}
$$

The power balance at each node is further modified, so that the positive and negative contributions of the line losses are accounted as additional load at the start and end buses. Typically, the positive component of a line loss is considered as a surplus of load at the upstream bus while the negative component is added to the load at the downstream bus ((9)). Finally, constraint (10) ensures that the line power and loss flow in the same direction.

$$
\begin{aligned}
& \left\{\begin{array}{l}
P_{b, t}=P_{b, t}^{L}+\delta p_{l_{u(b), t}^{+}}^{+}+\sum_{l_{d(b)} \in D(b)} \delta p_{l_{d(b), t}^{-}}^{-} \\
Q_{b, t}=Q_{b, t}^{L}+\delta q_{l_{u(b), t}^{+}}^{+}+\sum_{l_{d(b)} \in D(b)} \delta q_{l_{d(b)}, t}^{-}
\end{array}\right. \\
& \begin{cases}\delta p_{l, t}^{+} \leq p_{l, t}^{+}, \delta p_{l, t}^{-} \leq p_{l, t}^{-} \\
\delta q_{l, t}^{+} \leq q_{l, t}^{+}, \delta q_{l, t}^{-} \leq q_{l, t}^{-}\end{cases}
\end{aligned}
$$

\section{Losses Linearization}

This subsection focuses on the computation of the active and reactive losses introduced in the previous equations. Those losses are linearized following the typical piecewise 
decomposition [10]. The active and reactive branch flows are then represented by an additional set of positive variables, defined along the number of piece wise linear (PWL) segments. Following the linearization of the square function of a variable $x$ displayed in Fig. 3, based on [11], the PWL segments have the same length, equal to the expected maximum value of $x$ divided by the number of linear blocks (i.e. $K$ segments here). Constraint (11) then computes the overall line flows from each block contribution and considering the maximum power. Finally, the losses are estimated following (12) and (13) with the computation of the square function for both active and reactive flows in every possible direction. Note that if (8) allows to estimate the voltage drop, the loss estimations considers $V_{0}=1$ p.u., on the other hand, in order to avoid non linarites.

$$
\begin{aligned}
& \left\{\begin{array}{l}
p_{l, t}^{-}=\sum_{k \in K} p_{l, k, t}^{-}, p_{l, t}^{+}=\sum_{k \in K} p_{l, k, t}^{+}, 0 \leq p_{l, k, t}^{-}, p_{l, k, t}^{+} \leq \overline{p_{l}} / K \\
q_{l, t}^{-}=\sum_{k \in K} q_{l, k, t}^{-}, q_{l, t}^{+}=\sum_{k \in K} q_{l, k, t}^{+}, 0 \leq q_{l, k, t}^{-}, q_{l, k, t}^{+} \leq \overline{q_{l}} / K
\end{array}\right. \\
& \delta p_{l, t}^{+}+\delta p_{l, t}^{-}=\frac{r_{l}}{V_{0}^{2}} \cdot \sum_{k \in K}(2 \cdot k-1) \cdot\left(\frac{\overline{p_{l}} \cdot\left(p_{l, k, t}^{+}+p_{l, k, t}^{-}\right)}{K}+\frac{\overline{q_{l}} \cdot\left(q_{l, k, t}^{+}+q_{l, k, t}^{-}\right)}{K}\right) \\
& \delta q_{l, t}^{+}+\delta q_{l, t}^{-}=\frac{x_{l}}{V_{0}^{2}} \cdot \sum_{k \in K}(2 \cdot k-1) \cdot\left(\frac{\overline{p_{l}} \cdot\left(p_{l, k, t}^{+}+p_{l, k, t}^{-}\right)}{K}+\frac{\overline{q_{l} \cdot} \cdot\left(q_{l, k, t}^{+}+q_{l, k, t}^{-}\right)}{K}\right)
\end{aligned}
$$

One shortcoming of the considered linearization is that the obtained accuracy is highly impacted by the values chosen for the maximum line flows (both active and reactive), independently from the number of PWL blocks. Especially, the approximation of the square function is more accurate when the maximum expected flows are close to the actual values, which cannot be known a priori. Thus, this paper investigates two ways to set the values for the various $\bar{p}_{I}$, $\bar{q}_{l}$. At first, those values are set to a theoretical maximum line capacity that considers the sum of buses active/reactive loads penalized with a 'safety margin' of $50 \%$ (i.e. $\alpha=1.5$ in (14)). That approach denoted as $\mathbf{S}_{\mathbf{0}}$ then corresponds to a case in which all the feeders have the same rated capacity which is typically encountered in radial distribution networks operated in open loops.

$$
\overline{p_{l}}=\alpha \cdot \sum_{b \in B} \max \left(P_{b, t}^{L}\right) \text { and } \overline{q_{l}}=\alpha \cdot \sum_{b \in B} \max \left(Q_{b, t}^{L}\right) \forall t \in T
$$

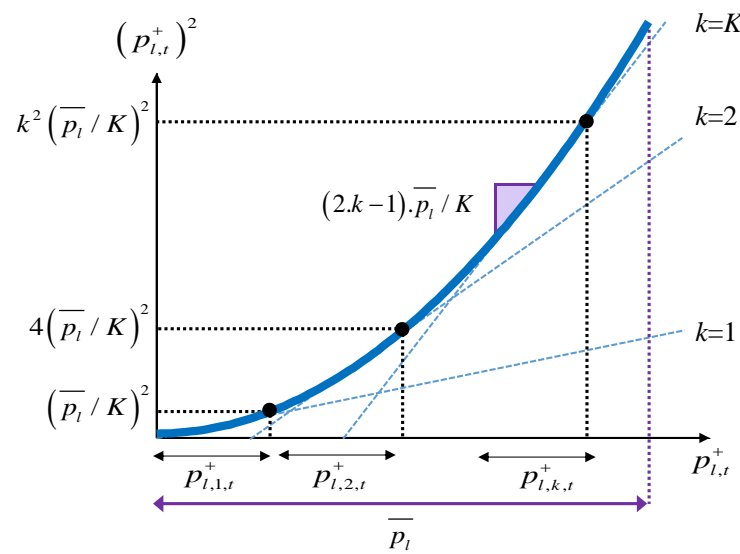

Fig. 3 Piewise linearization of quadratic functions applied to the line flow [11]
ALGO1 Computation of LiLne Limit Flows in $\mathbf{S}_{\mathbf{1}}$

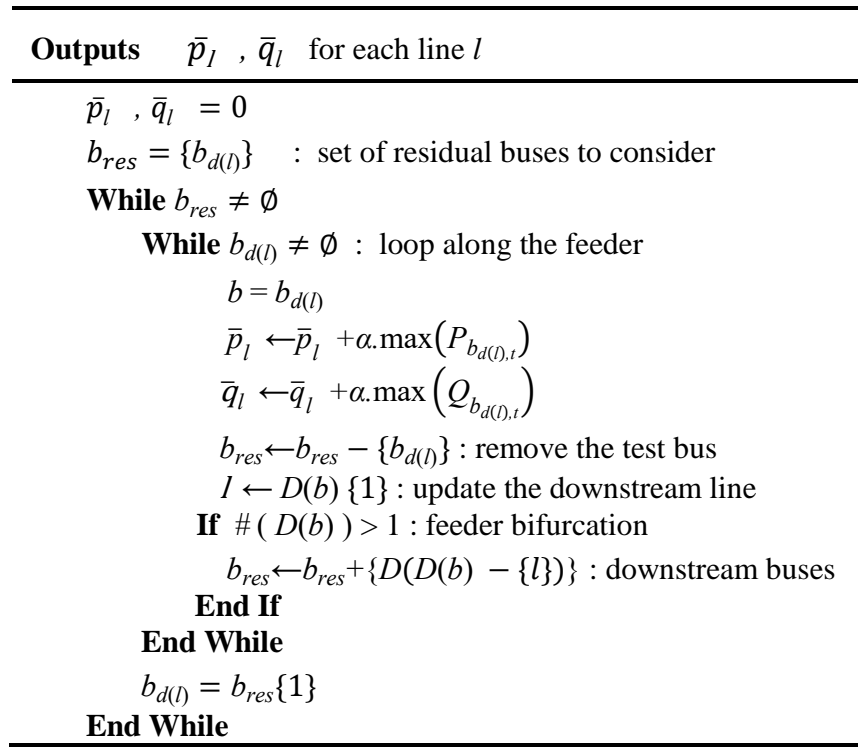

An alternative proposed here is to set the branch maximum flows $\bar{p}_{l}, \bar{q}_{l}$ closer to the expected actual value. That approach denoted as $\mathbf{S}_{\mathbf{1}}$, consists in doing a graph search, i.e. considering the active and reactive loads at all the buses located downstream each line while paying attention to potential feeder bifurcation (refer to Algo I with \# denoting the cardinal function). As previously, the expected maximum flows are affected by a safety margin sufficient enough to guarantee the convergence of the power flow formulation (i.e. the actual line flows are expected to be within the specified boundaries with the given distribution of the bus load/injection).

\section{Validation Runs}

The previous equations are entered as constraints of an optimization problem that represents the practical implementation of the considered power flow. To be consistent with typical OPF, the objective to minimize is the total system losses, indiscriminately on the active and reactive components (in per unit in (15)). The corresponding linear problem is written using YALMIP toolkit [12] and solved with CPLEX 12.10.0 (8 GB RAM, 1.6 GHz processor).

$$
\text { obj: } \min \sum_{t \in T} \sum_{l \in L} \delta p_{l, t}(\text { p.u. })+\delta q_{l, t}(\text { p.u. })
$$

At first, the modified DistFlow is developed and tested on a simple 7 buses system displayed in Fig. 4, before being scaled to $18,33,69$ and 85 nodes networks, and while considering only a snapshot for the bus load (i.e. single element in the temporal set $T$ ). Also, only the import from the upstream grid is considered at first with no distributed resources connected to the considered systems. The results obtained are compared with the outputs of a conventional ACPF run with MATPOWER. Especially, three error criteria are defined to estimate the deviation in the voltages bus, active and reactive losses with regard to the ACPF results (denoted with the upper script ${ }^{\mathrm{AC}}$ ). Those errors are normalized with regard to the mean deviation of the voltage computed over the total number of buses and the mean losses estimated over all the branches ((16)). 
a)

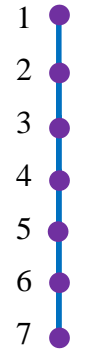

Fig. 47 buses systems $-a) 7$ bus $\mathrm{A}-b) 7$ bus $\mathrm{B}$

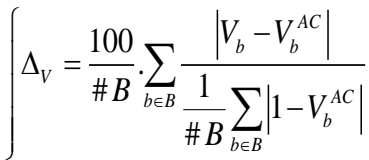

$$
\begin{aligned}
& \Delta_{\delta P}=\frac{100}{\# L} \cdot \sum_{l \in L} \frac{\left|\delta p_{l}-\delta p_{l}^{A C}\right|}{\# L} \sum_{l \in L} \delta p_{l}^{A C}, \Delta_{\delta Q}=\frac{100}{\# L} \cdot \sum_{l \in L} \frac{\left|\delta q_{l}-\delta q_{l}^{A C}\right|}{\# L \sum_{l \in L} \delta q_{l}^{A C}}
\end{aligned}
$$

Table I presents the results obtained for the different validation runs. Tests with the 7 buses systems show an increased model accuracy with greater numbers of PWL segments. Note that the error for the active and reactive line losses are equal for the case study as the $x_{l} / r_{l}$ ratio is constant for all the branches. For the other test cases, the best performances ( $5 \%$ error on average) are returned with $K=2$ and while estimating the branches maximum flows considering the aggregated load at the downstream buses for every line. The great improvements (error divided by ten) observed when using $\mathbf{S}_{\mathbf{1}}$ highlight the impact of the $\bar{p}_{l}, \bar{q}_{l}$ in the considered linearization method. That approach should be furtherly investigated while adapting the 'safety margin' $\alpha$, or considering other intputs for the branch expected maximum flow (e.g. DC power flow results).

TABLE I. RESULTS OF VALIDATION RUNS

\begin{tabular}{c|ccc|ccc} 
& \multicolumn{2}{c}{ Maximum line flow $\mathbf{S}_{\mathbf{0}}$} & \multicolumn{3}{c}{ Maximum line flow $\mathbf{S}_{\mathbf{1}}$} \\
\cline { 2 - 7 } & $K=1$ & $K=2$ & $K=3$ & $K=1$ & $K=2$ & $K=3$ \\
\hline $\mathbf{7}$ bus A & & & & & & \\
$\Delta_{V}(\%)$ & 4.5 & 0.9 & 0.5 & 1.8 & 0.4 & $\mathbf{0 . 3}$ \\
$\Delta_{\delta P}(\%)$ & 106.6 & 22.1 & 9.38 & 44.5 & 9.1 & $\mathbf{2 . 8}$ \\
$\Delta_{\delta Q}(\%)$ & 106.6 & 22.1 & 9.38 & 44.5 & 9.1 & $\mathbf{2 . 8}$ \\
$\mathbf{7}$ bus B & & & & & & \\
$\Delta_{V}(\%)$ & 8.3 & 2.6 & 1.1 & 2.4 & 0.6 & $\mathbf{0 . 3}$ \\
$\Delta_{\delta P}(\%)$ & 130.7 & 37.3 & 12.4 & 42.3 & 7.4 & $\mathbf{4 . 2}$ \\
$\Delta_{\delta Q}(\%)$ & 130.7 & 37.3 & 12.4 & 42.3 & 7.4 & $\mathbf{4 . 2}$ \\
& & & & & & \\
$\Delta_{V}(\%)$ & 39.5 & 6.9 & 3 & 3.2 & $\mathbf{2}$ & 3.2 \\
$\Delta_{\delta P}(\%)$ & 437.4 & 161.7 & 73.3 & 19.3 & $\mathbf{9 . 5}$ & 18.7 \\
$\Delta_{\delta Q}(\%)$ & 198.7 & 188.1 & 18.4 & 22.6 & $\mathbf{6 . 1 5}$ & 15.4 \\
$\mathbf{3 3}$ bus & & & & & & \\
$\Delta_{V}(\%)$ & 23.15 & 6.9 & 2.2 & $\mathbf{1 . 7 5}$ & 2.8 & 3.2 \\
$\Delta_{\delta P}(\%)$ & 462.5 & 161.8 & 80.4 & 35.9 & $\mathbf{5 . 3}$ & 6.9 \\
$\Delta_{\delta Q}(\%)$ & 519 & 188.1 & 95.4 & 35.1 & $\mathbf{4 . 7}$ & 7.7 \\
$\mathbf{6 9}$ bus & & & & & & \\
$\Delta_{V}(\%)$ & 19.5 & 5.5 & 2.2 & $\mathbf{1 . 2}$ & 2.2 & 2.7 \\
$\Delta_{\delta P}(\%)$ & 414.9 & 139.6 & 63.5 & 35.6 & $\mathbf{5 . 2}$ & 8.2 \\
$\Delta_{\delta Q}(\%)$ & 425.6 & 145.3 & 68.8 & 35.1 & $\mathbf{6 . 1}$ & 7 \\
$\mathbf{8 5}$ bus & & & & & & \\
$\Delta_{V}(\%)$ & 26.15 & 6.2 & $\mathbf{1 . 4}$ & 2.9 & 4.6 & 5.3 \\
$\Delta_{\delta P}(\%)$ & 377.3 & 118.9 & 66.8 & 27.3 & $\mathbf{6 . 5}$ & 11.8 \\
$\Delta_{\delta Q}(\%)$ & 316.6 & 90.2 & 50.3 & 28 & $\mathbf{6 . 2}$ & 11.1 \\
\hline
\end{tabular}
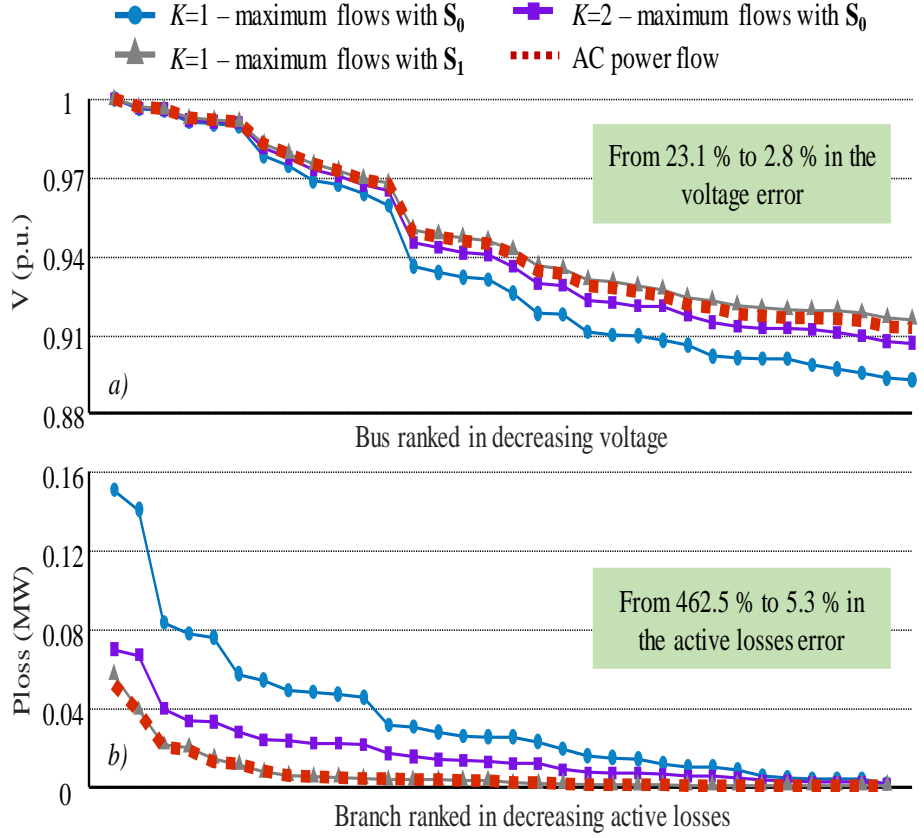

Fig. 5 Validation runs for 33 bus test case $-a$ ) bus voltage $-b$ ) active losses

Fig. 5 displays the performances of the modified DistFlow applied to the 33 bus test case. Especially, different settings are considered in order to highlight the successive improvements (both in terms of losses and voltage errors) while increasing the number of PWL segments, and then computing the maximum line flow according to $\mathbf{S}_{\mathbf{1}}$. Another noticeable results of Fig. 5 is that the rank of the buses with the greatest voltage drop and the branches with the greatest losses is the same when considering the reference ACPF and the modified DistFlow introduced in the paper. It suggests that, in the context of an optimal design, the DistFlow appropriately discriminates the best allocations of the distributed resource, even though the estimation of the losses and voltage may not be the most accurate.

\section{TESt CAse For ResourCES Alocation}

\section{A. DG Alocation Problem Formulation}

In this section, a generic, simple DG allocation problem is considered in order to investigate the computational performances of the proposed method. The IEEE 69 buses radial test case is considered (Fig. 6) and the objective of the study is to optimally determine the best site for a DG consisting of $500 \mathrm{kWp}$ of solar panels and a $500 \mathrm{~kW} / 500 \mathrm{kWh}$ battery. The implemented DistFlow is then run every time a new site is investigated in order to simulate the system operation over a representative period. A single day is considered here with hourly profiles for the solar radiation as well as for the different types of loads (i.e. industrial, commercial and residential for a total of 48 distinct patterns). With given profiles for the load and generation, the only degree of freedom of the system is the scheduling of the storage over the simulated period. The problem formulation described in the previous section is then appended to integrate the operating constraints for the battery. A typical linear formulation is used while discriminating the charge and discharge powers of the battery to compute the state of charge with a given efficiency [13]. The set of constraints (17) then ensures the fulfilment of the power/energy limitations and 
enforces any storage device to come back at its initial state of charge SOC at the end of the simulated day (typically $50 \%$ ).

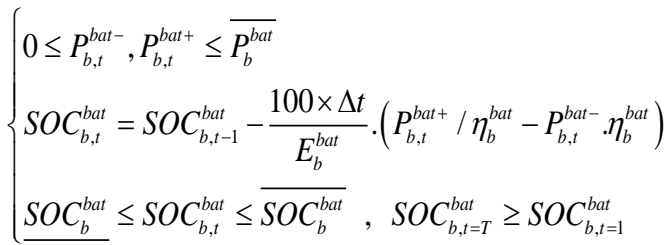

In addition, the active power balance at each bus is modified following (18) in order to take the solar and battery injections into account. A single solar/storage installation is considered. Thus, the battery variables and parameters for the PV generation are only considered for the connection bus, with the use of appropriate matrixes not described in this paper. The objective of the allocation problem is to reduce the system losses. The objective (15) remains unchanged with the minimization of the system losses, consisting not only of the grid effects but also of the storage loses implicitly captured in (18). Ultimately the optimal scheduling problem is run over a representative day with 25,000 variables and a little more than 50,000 linear constraints for an average computational time of 1 to $2 \mathrm{~s}$.

$$
P_{b, t}=P_{b, t}^{L}-P_{b, t}^{P V}-P_{b, t}^{b a t+}+P_{b, t}^{b a t-}+\delta p_{l_{u(b), t}^{+}}^{+}+\sum_{l_{d(b)} \in D(b)} \delta p_{l_{d(b)}^{-}, t}^{-}
$$

\section{B. Obtained Resuls}

The optimization method in charge of determining the best location is not in the scope of the paper. Typically, evolutionary or heuristic approaches or heuristics are considered in order to investigate the best potential sites. With the fast computation allowed by the implemented DistFlow, an exhaustive search investigates the connection of the PV/battery system at every node of the 69 buses system in less than 2 minutes. The operation of the battery is then optimized in every case over the simulated day and the system losses are compared to a base case in which no distributed resources are installed (i.e. daily load supplied by the upstream grid only). Fig. 6 displays the obtained results with three areas defined according to the loss reduction compared to the base case scenario. Greater efficiencies are reached when the resources tend to be installed at the end of the longest feeder (3-6\%) and closer to the most significant point of consumption (i.e. the industrial load here) with more than $8 \%$ loss reduction for the simulated day.

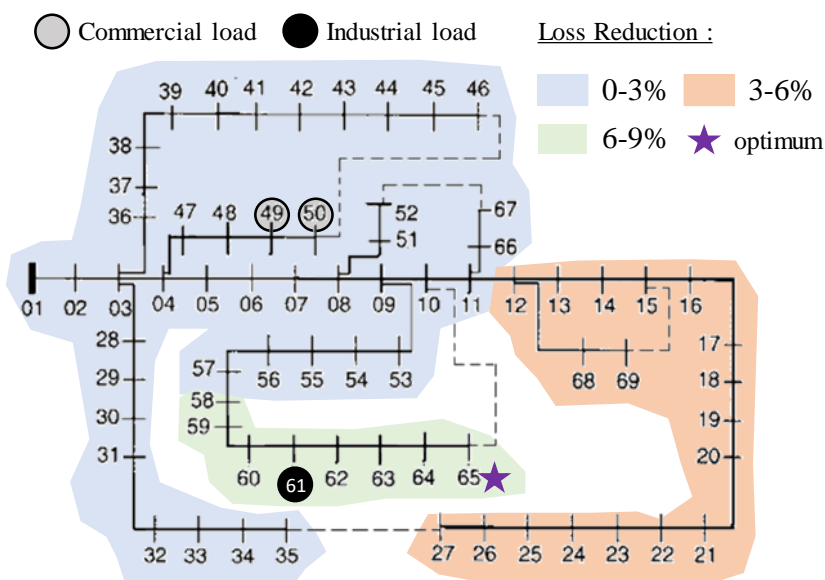

Fig. 6 Considered 69 bus system and allocation results
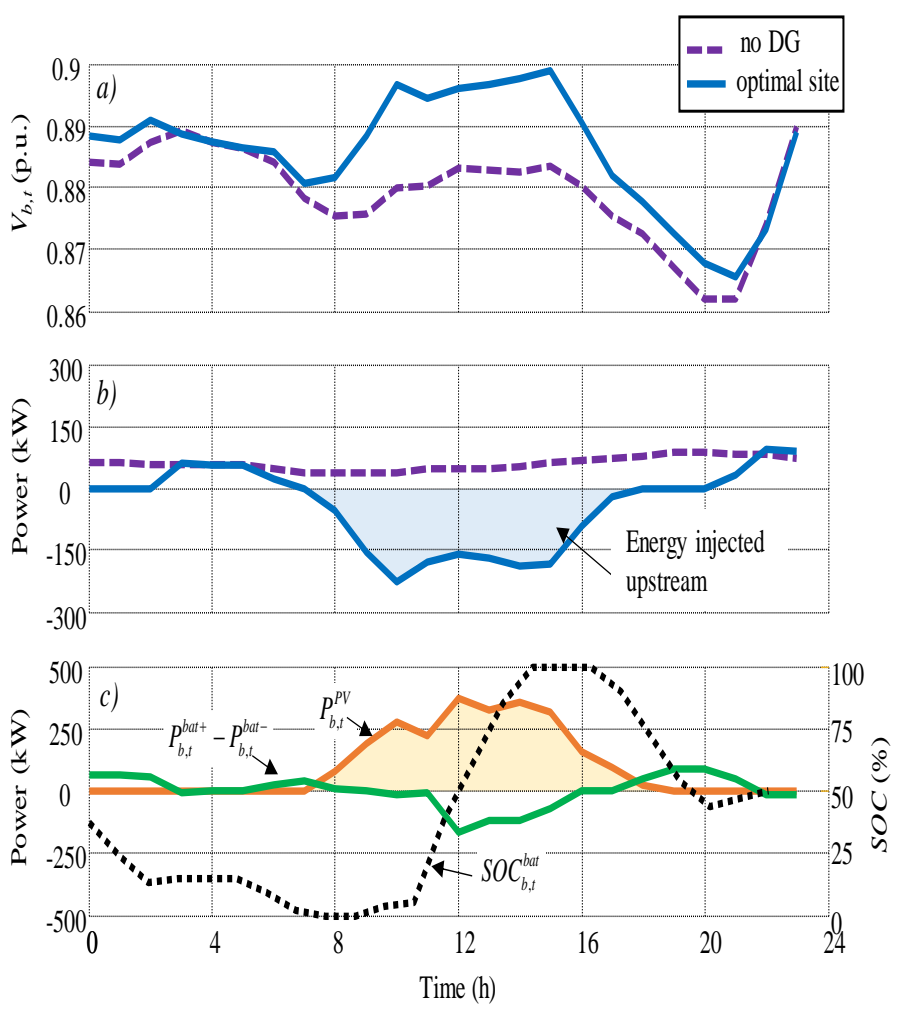

Fig. 7 Results at bus $21-a$ ) bus voltage $-b$ ) power at the incident branch c) power injections and battery $S O C$

It is important to note that the estimated losses reduction are mostly (> $75 \%$ ) allowed by the integration of the solar generation. Indeed, the storage does not participate that much to the savings due to its internal losses and the energy conservation constraint (final SOC). The loss reduction allowed by the storage corresponds to the peak shaving of the total injected power. As mentioned, the best configuration is obtained with the installation connected to bus 65 , at the end of the feeder hosting the heaviest customer. Especially, the solar generation during the daily hours tends to decreases the voltage drop by more than 0.01 p.u. compared to the base case scenario (Fig. 7a). With the significant amount of locally injected energy, the power flowing through the incident branch (i.e. from bus 64 to bus 65 ) takes negative values during the sun peak hours (i.e. reverse power) as observed on Fig. $7 b$. Without any consideration of energy prices or battery degradation, the optimal operation of the storage follows the dynamics of the solar generation respectively to the load profiles. Part of the solar is locally self-consumed to charge the battery during the day while the morning and evening peak load tends to be lowered with appropriate discharge rates of the storage (Fig. 7c).

\section{Scaliability and Computational Performances}

The implemented solution then allows fast computation and the solution is quickly found with the simple validation study considered above. However, problems encountered in the literature and at the grid planner level may display higher degree of complexity: wider systems in terms of nodes, longer planning horizons, finer time resolutions, greater numbers of distributed resources, uncertain parameters, non linearities, etc... Thus, a last set of runs is performed in order to test the scalability of the proposed approach. As the full spectrum of complexity cannot be investigated, the authors 
focus here on the duration of the simulated period as well as the numbers of installed resources. The number of time steps $T$ is then increase from 24 (i.e. one day here at $1 \mathrm{~h}$ resolution) to 2160 (i.e. 3 months), while the number of installed DG varies up to fifty units. A maximum computational time of 5 min arbitrarily chosen. As already mentioned, the solving duration of the system operation should not be too significant as several runs are likely to be executed for optimal DG placement problems. Table II displays the results obtained with "N.A." in case the time limit is exceeded. For the considered 69 buses system the modified DistFlow allows to compute up to 3 months at an hourly resolution and with 10 different DG connected at 10 different buses. It is worth mentioning that the DG assets are assumed to be controlled in a centralized fashion here, which might not be the case in actual deployment due to technical and regulatory limitations. The main objective here is merely to test the mathematical scalability of the proposed approach.

TABLE II. COMPUTATIONAL TIMES FOR SCALABILITY TEST

\begin{tabular}{c|c|c|c|c|c|c}
\hline TG & $\mathbf{1}$ & $\mathbf{7}$ & $\mathbf{1 5}$ & $\mathbf{3 0}$ & $\mathbf{6 0}$ & $\mathbf{9 0}$ \\
\hline $\mathbf{1}$ & $2 \mathrm{~s}$ & $12 \mathrm{~s}$ & $30 \mathrm{~s}$ & $70 \mathrm{~s}$ & $150 \mathrm{~s}$ & $217 \mathrm{~s}$ \\
$\mathbf{1 0}$ & $2 \mathrm{~s}$ & $12 \mathrm{~s}$ & $30 \mathrm{~s}$ & $85 \mathrm{~s}$ & $210 \mathrm{~s}$ & $290 \mathrm{~s}$ \\
$\mathbf{2 0}$ & $2 \mathrm{~s}$ & $15 \mathrm{~s}$ & $40 \mathrm{~s}$ & $90 \mathrm{~s}$ & $250 \mathrm{~s}$ & N.A. \\
$\mathbf{3 0}$ & $2 \mathrm{~s}$ & $21 \mathrm{~s}$ & $50 \mathrm{~s}$ & $130 \mathrm{~s}$ & N.A. & N.A. \\
$\mathbf{4 0}$ & $3 \mathrm{~s}$ & $50 \mathrm{~s}$ & $110 \mathrm{~s}$ & $130 \mathrm{~s}$ & N.A. & N.A. \\
$\mathbf{5 0}$ & $3 \mathrm{~s}$ & $60 \mathrm{~s}$ & $130 \mathrm{~s}$ & N.A. & N.A. & N.A. \\
\hline
\end{tabular}

Globally, with the computational capabilities considered in the study, the time limit to solve the fully linear problem is reached for around 2 million variables or more. That number of variables depends on the size of the system ( $B$ buses), the time horizon ( $T$ time steps) as well as the number of DG resources denoted as $N$. Finally (19) allows to plot an expected feasible region (Fig. 8) that represents the size of the problem with an acceptable solving time. In theory the developed method would allow to simulate a monthly operation of a 100 buses system with a hundred connected DG.

$T \cdot(15 . B+3 . N) \leq 2.10^{6}$

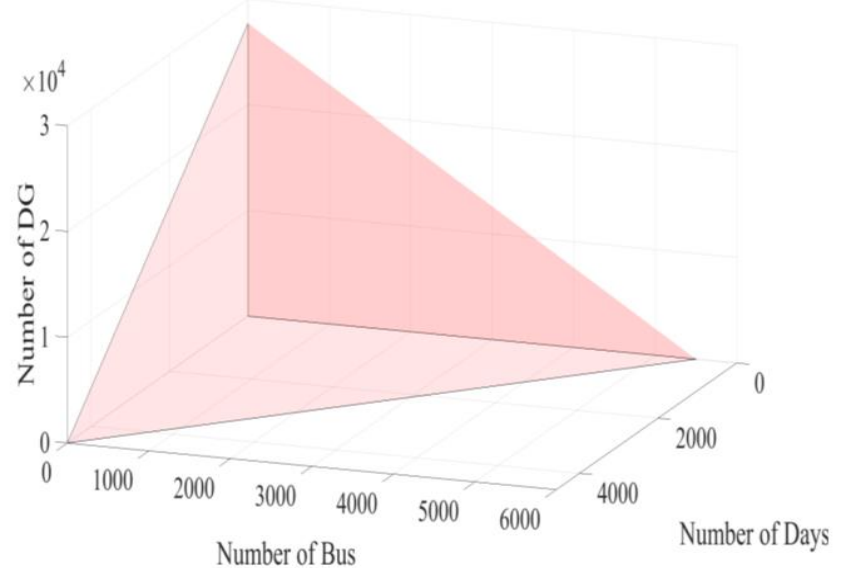

Fig. 8 Feasible region with a solving time lower or equal at $5 \mathrm{~min}$

\section{CONCLUSIONS}

This paper successfully implements a linearized DistFlow that intent to be used in grid simulation for DG planning studies. Compared to conventional linearized approaches, the formulation presented here allows to consider the effects of the losses on the voltage drop and discriminates the flow direction of both active and reactive powers. The method is validated and tested on various systems before a generic DG planning problem is solved on a 69 buses network in less than 2 minutes with an exhaustive search. Scalability tests of the implemented linear algorithm show its ability to solve more complex problems on longer time horizons. Future works consist in taking advantage of that fast computation to investigate more advanced planning strategies, multi objectives approaches, different types of DG or more complex control modes.

\section{REFERENCES}

[1] A.R. Jordehi, "Allocation of distributed generation units in electric power systems: a review", Renew Sustain Energy Rev., vol. 56, pp. 893-905, Apr. 2016.

[2] P.Paliwal, N.P. Patidar, R.K. Nema, "Planning of grid integrated distributed generators:a review of technology, objectives and techniques", Renew Sustain Energy Rev, vol. 40, pp. 557-570, Dec. 2014.

[3] R. Rigo-Mariani, K.V. Ling, J. Maciejowski, “A clusterized energy management with linearized losses in the presence of multiple types of distributed generation", Electrical Power and Energy Systems, vol. 113 pp 9-22, May 2019.

[4] L.F. Ochoa, C.J. Den, G.P. Harrison, "Distribution network capacity assessment: variable DG and Active networks", IEEE Trans Power Syst; vol. 25, no. 1, pp 87-95, Nov. 2009.

[5] R. Villasana, L.L. Garve, S.J. Salon, “ Transmission Network Using Linear Programming", IEEE Trans. Power Apparatus and Systems, vol. 104, no. 2, Feb. 2015

[6] M. E. Baran and F. F.Wu, "Optimal capacitor placement on radial distribution systems," IEEE Trans. Power Del., vol. 4, no. 1, pp. 725734, Jan. 1989.

[7] K. Turitsyn, P. Sulc, S. Backhaus, M. Chertkov, "Distributed Control of Reactive Power Flow in a Radial Distribution Circuit with High Photovoltaic Penetration", IEEE PES Genral Meering, Providence, RI, USA, Jul. 2010.

[8] Z. Wang, B. Chen, J. Wang, J. Kim, "Decentralized Energy Management System for Networked Microgrids in Grid-Connected and Islanded Modes", IEEE Trans. Smart Grid, vol. 7, no. 2, pp. 10971105, Mar. 2016.

[9] H.G. Yeh, D.F. Gayme, S.H. Low, "Adaptive VAR Control for Distribution Circuits With Photovoltaic Generators", IEEE Trans. Power Systems, vol. 27, no. 3, pp. 1656-1663, Aug. 2012.

[10] M. Carrión, J. M. Arroyo, "A computationally efficient mixed-integer linear formulation for the thermal unit commitment problem", IEEE Trans. On Power Systems, vol 21, no. 3, pp 1371-1378, 2006.

[11] P. L. Cavalcante, J. Camilo López, J. F. Franco, M. J. Rider, A.V. Garcia, M.R.R. Malveira, L.L. Martins, L. C. M. Direito, "Centralized Self-Healing Scheme for Electrical Distribution Systems”, IEEE Trans. Smart Grid, vol. 7, no. 1, pp. 145-155, Aug. 2015.

[12] J. Lofberg, "YALMIP: a toolbox for modelling and optimization in MATLAB", IEEE International Symposium on Computer Aided Control Systems Design, New-Orleans, USA,, pp. 282-289, 2004

[13] H. Pandžić, Y. Wang, T. Qiu, Y. Dvorkin, D.S. Kirschen, "Nearoptimal method for siting and sizing of distributed storage in a transmission network", IEEE Trans Power Syst., vol. 25, no. 1, pp. 2288-3000, pp. 2288-2300, Oct. 2014. 